\title{
Interpenetrating polymer networks of 2-hydroxyethyl methacrylate terminated polyurethanes and polyurethanes
}

\author{
Tsung-Tang Hsieh ${ }^{\mathrm{a}, *}$, Kuo-Huang Hsieh ${ }^{\mathrm{b}}$, George P. Simon ${ }^{\mathrm{c}}$, Carlos Tiu ${ }^{\mathrm{a}}$ \\ ${ }^{a}$ Department of Chemical Engineering, Monash University, Clayton, Victoria 3168, Australia \\ ${ }^{\mathrm{b}}$ Department of Chemical Engineering, National Taiwan University, Taipei 106, Taiwan, ROC \\ ${ }^{\mathrm{c}}$ Department of Materials Engineering, Monash University, Clayton, Victoria 3168, Australia
}

Received 18 August 1997; revised 16 January 1998; accepted 3 March 1998

\begin{abstract}
Interpenetrating polymer networks (IPNs) of 2-hydroxyethyl methacrylate terminated polyurethanes (HPU) and polyurethanes (PU) with different ether-type polyols were prepared by simultaneous solution polymerization. Differential scanning calorimetry showed that the compatibility of polymers in IPN formation depends on the molecular weight of the prepolymers. Compatible IPN systems resulted in greater-than-average density values and reduced swelling behaviour. Water absorption of the IPNs was found to be mainly determined by the hard-to-soft segment ratio of the materials. The maximum tensile modulus occurred at a $25 \mathrm{wt} \%$ HPU content for the compatible systems and a $12.5 \mathrm{wt} \%$ HPU content for those that were incompatible. The maximum tensile strength occurred at a $25 \mathrm{wt} \%$ HPU content for all the IPN systems. Morphological observations using a scanning electron microscope revealed different fracture surfaces between the compatible and incompatible systems. (C) 1999 Elsevier Science Ltd. All rights reserved.
\end{abstract}

Keywords: Interpenetrating polymer networks; Polyurethanes; Compatibility

\section{Introduction}

Interpenetrating polymer networks (IPNs) constitute a rapidly developing branch of polymer blend technology which may intimately combine the properties of two crosslinked polymers in a network form. By definition, an IPN structure is obtained when at least one polymer network is synthesized and/or crosslinked independently in the immediate presence of the other [1]. As long as the reacting ingredients are mixed well during synthesis, thermodynamic incompatibility can be overcome due to permanent interlocking of network segments and IPNs with limited phase separation are obtained. It has been reported that polymeric materials with improved mechanical properties can be obtained via IPN structures [2-5]. This synergistic effect can be attributed to an increase in the crosslinking density where two networks are formed by the interpenetration of each other [6,7]. Suggested applications of IPNs have included their use as damping materials [8-10], biomedical materials [10,11], gas transport membranes [12,13], and electrical/electronic devices [14].

Polyurethanes (PUs) comprise a class of materials which

\footnotetext{
* Corresponding author. Tel: + 61-39905-1482; Fax: + 61-399055686; e-mail: hsieh@its-engl.monash.edu.au
}

can vary widely from rubbery to glassy thermoplastics and from being linear polymers to being a thermoset. This versatility can be further controlled in terms of processing and composition to fulfil a vast variety of product requirements. If the component compounds contain isocyanate groups (polyisocyanates) and include active hydrogen atoms (polyethers, polyesters, etc.), the processing conditions can be varied and the properties of PUs can be tailor-made to produce desirable molecular structures and physical properties [15-17]. As a result of their high mechanical strength, flexibility, fatigue resistance and biocompatible nature, PUs have been proven to be potential candidates as materials for artificial organs [18-20].

In this study, IPNs of two types of PUs were synthesized and their properties investigated. As these IPNs were aimed at biomedical applications [21], ether-type polyols were chosen to increase their resistance to hydrolytic degradation and to ensure biocompatibility. Component 1 of the IPNs was a PU based on an isocyanate-terminated PU prepolymer with an equivalent ratio of MDI:polyol of 3:1 and crosslinked by a triol. In order to introduce a PU formation mechanism other than general diisocyanate polyaddition, PU prepolymers with terminal isocyanate groups were end-capped by HEMA to form one- component-type PU prepolymers or UV-curable urethane prepolymers $[22,23]$. 
Table 1

Materials used in this work

\begin{tabular}{|c|c|c|}
\hline Designation & Description & Source \\
\hline PTMG1000 & $\begin{array}{l}\text { Poly(tetramethylene oxide) } \\
\text { glycol, } M_{\mathrm{w}}=1000\end{array}$ & Chium Glong Co. \\
\hline PTMG2000 & $\begin{array}{l}\text { Poly(tetramethylene oxide) } \\
\text { glycol, } M_{\mathrm{w}}=2000\end{array}$ & Chium Glong Co. \\
\hline PPG1000 & $\begin{array}{l}\text { Poly(propylene oxide) } \\
\text { glycol, } M_{\mathrm{w}}=1000\end{array}$ & Chium Glong Co \\
\hline PPG2000 & $\begin{array}{l}\text { Poly(propylene oxide) } \\
\text { glycol, } M_{\mathrm{w}}=2000\end{array}$ & Chium Glong Co. \\
\hline MDI & $\begin{array}{l}4,4^{\prime \prime} \text {-Diphenylmethane } \\
\text { diisocyanate }\end{array}$ & Tai Chin Co. \\
\hline HEMA & 2-Hydoxyethyl methacrylate & Merck Chemical Co. \\
\hline 1,4-BD & 1,4-Butanediol & Hayashi Pure Chem. \\
\hline TMP & 1,1,1-Trimethylol propane & Hayashi Pure Chem. \\
\hline BPO & Benzoyl peroxide & Merck Chemical Co. \\
\hline DMF & $N, N^{\prime \prime}$-Dimethylformamide & Merck Chemical Co. \\
\hline DBTDL (T-12) & Dibutyltin dilaurate & Merck Co. \\
\hline
\end{tabular}

A HEMA-terminated PU (HPU) prepolymer with an equivalent ratio of MDI:polyol of 2:1, as component 2 of the IPNs, possessed a double bond at each chain end and is thus capable of forming a network structure via radical polymerization. Simultaneous solution polymerization was adapted to prepare the IPN samples so that better mixing of these two kinds of long-chain prepolymers (both with molecular weights of around 1500-2500) could be accomplished. The formation of allophanates is believed to be negligible compared to the formation of the urethanes from which they are derived because of the much lower reactivity of the active hydrogen atoms in urethanes at the reaction temperature $\left(70^{\circ} \mathrm{C}\right)$ and the use of a catalyst $(\mathrm{T}-12)$ to accelerate the primary isocyanate/hydroxyl group reaction in the current study [15-17]. The influence of different reaction mechanisms and molecular weights of the polyols on the physical properties of these HPU/PU IPNs is discussed.

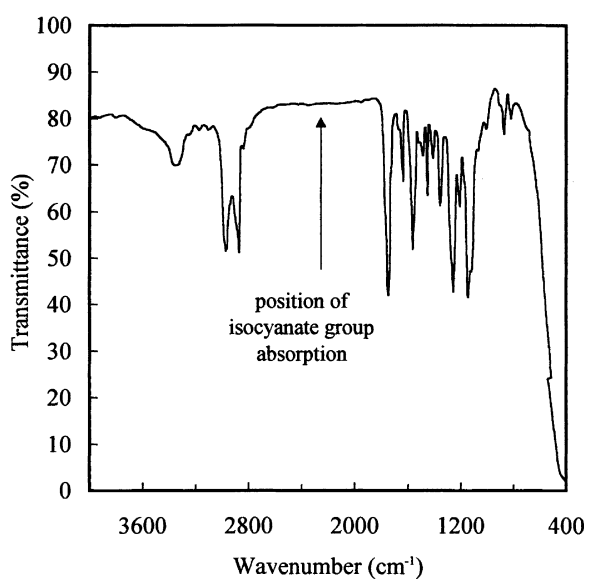

Fig. 1. Infrared spectrum of the HPU(PTMG1000) prepolymer at the end of the reaction. No absorption of the isocyanate group $\left(2270 \mathrm{~cm}^{-1}\right)$ is detected.

\section{Methods}

\subsection{Materials}

The raw materials and reagents employed and their details are given in Table 1. MDI was melted in an oven at $55^{\circ} \mathrm{C}$ and the upper-layer of clear liquid was used for the reaction. The equivalent weight of the received MDI was determined by means of the di- $n$-butylamine titration method [16] before use. Characterization of the polyols (PPG1000, PPG2000, PTMG1000 and PTMG2000) was carried out by gel permeation chromatography (g.p.c.) and hydroxyl group number determination. In order to remove any moisture, the ether-type polyols, 1,4-BD (chain extender) and TMP (crosslinking agent) were stirred and degassed in the flasks at $70^{\circ} \mathrm{C}$ under reduced pressure for $16 \mathrm{~h}$. DMF and HEMA were dried for at least 1 week using $4 \AA$ molecular sieve before use.

\subsection{Preparation of $H P U / P U I P N s$}

In the preparation of the PU prepolymer, three equivalents of MDI and one equivalent of PPG were charged in a four-necked reaction vessel equipped with a mechanical stirrer. The reaction was carried out at $70^{\circ} \mathrm{C}$ under a dry nitrogen atmosphere. When the isocyanate group content (determined by the di- $n$-butylamine titration method [16]) of the reaction mixture reached the theoretical value (all PPG molecules end-capped by MDI) the reaction was terminated. The unreacted free MDI was left in the synthesized PU prepolymer to give the PU network a higher hard-to-soft segment ratio.

The HPU prepolymer was prepared in two steps. First, two equivalents of MDI and one equivalent of PTMG were added to a reaction vessel. A catalyst, T-12, was added to accelerate the reaction. The mixture was agitated and allowed to react for $30 \mathrm{~min}$. An equivalent of HEMA was then added by syringe and mixed. The reaction proceeded until the infrared absorption of the isocyanate group $\left(2270 \mathrm{~cm}^{-1}\right)$ of the sample taken disappeared, as shown in Fig. 1.

For the synthesis of HPU/PU IPNs, suitable amounts of PU prepolymer, HPU prepolymer, the 1,4-BD/TMP mixture, T-12 and BPO (at a concentration of $2 \mathrm{wt} \%$ of HPU prepolymer) were dissolved in DMF to form a $40 \mathrm{wt} \%$ solution. The reaction was maintained at $70^{\circ} \mathrm{C}$ in a reaction vessel under a dry nitrogen purge for $40 \mathrm{~min}$. The viscous solution was degassed to remove trapped bubbles and cast into a glass dish which was then left for further reaction and the vaporization of solvent in an oven at $70^{\circ} \mathrm{C}$ for a further $24 \mathrm{~h}$. The membrane formed was post-cured at $110^{\circ} \mathrm{C}$ for $12 \mathrm{~h}$ and released from the glass dish. Before use, all samples were vacuum dried for $24 \mathrm{~h}$ at $70^{\circ} \mathrm{C}$. The synthesis processes for the production of PU prepolymer, HPU prepolymer and their IPNs are shown in Fig. 2. 

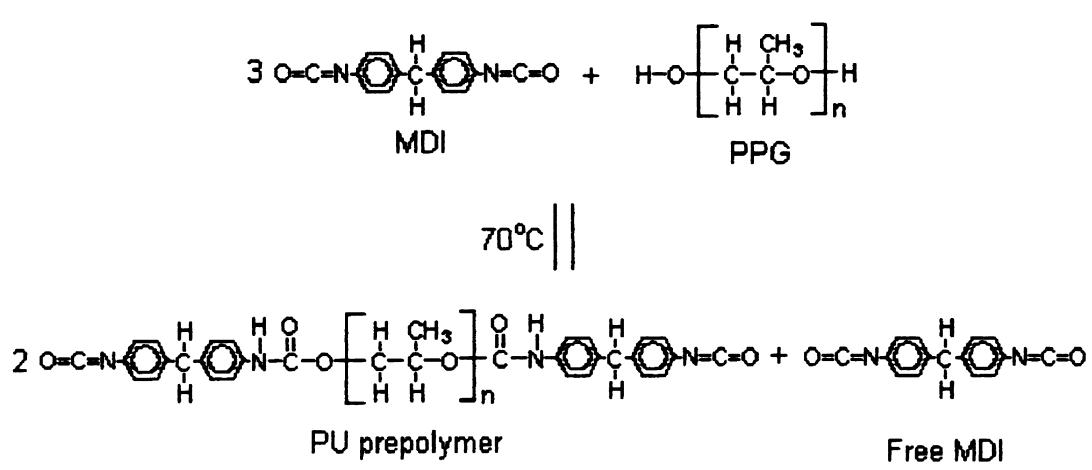

(a)

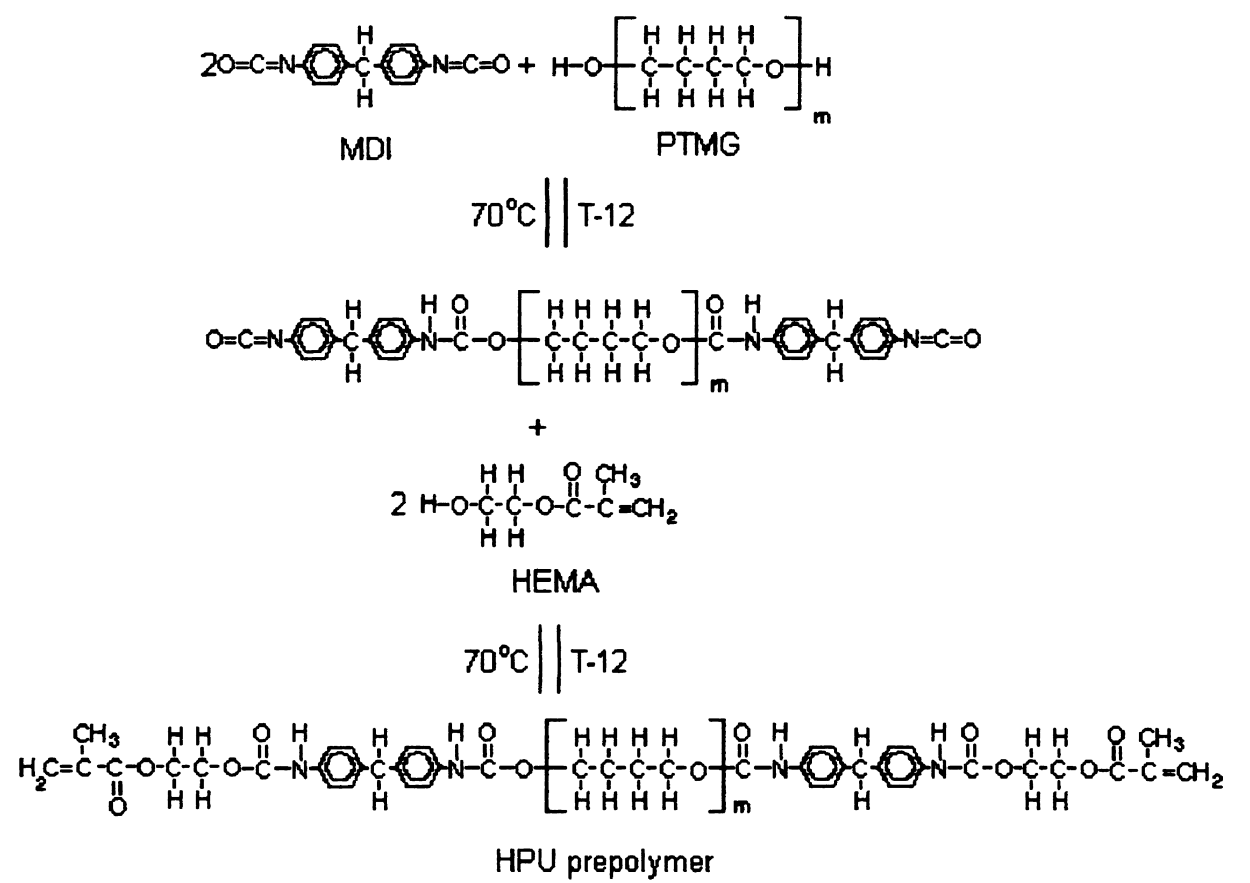

(b)

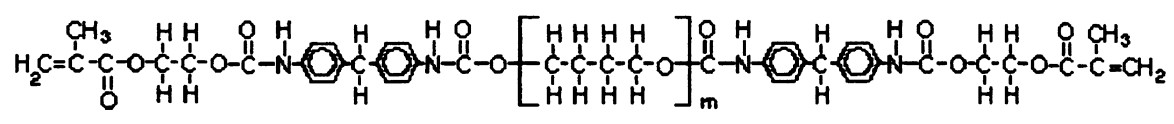
HPU prepolymer

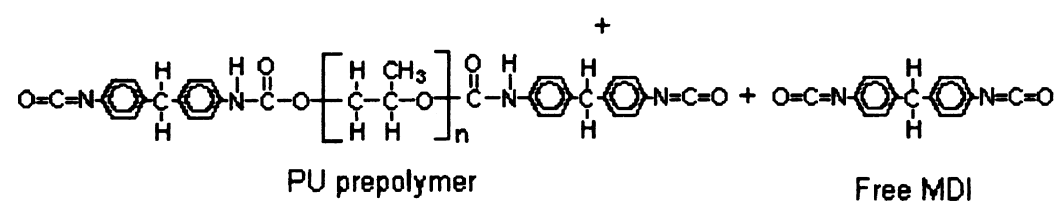

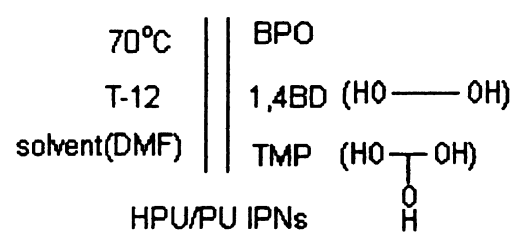

(c)

Fig. 2. Synthesis process of (a) PU prepolymer, (b) HPU prepolymer, and (c) HPU/PU IPNs. 


\subsection{Testing methods}

The thermal properties of the IPNs were measured with use of a Du Pont 9900-910 differential scanning calorimeter in a nitrogen atmosphere. All samples of 15-20 mg were initially scanned over the temperature range from -120 to $150^{\circ} \mathrm{C}$ at a heating rate of $10^{\circ} \mathrm{C} \mathrm{min}^{-1}$ and quenched to $-120^{\circ} \mathrm{C}$ as rapidly as possible (at a cooling rate of $100^{\circ} \mathrm{C} \mathrm{min}{ }^{-1}$ from $150^{\circ} \mathrm{C}$ down to $20^{\circ} \mathrm{C}$ and a cooling rate of $10^{\circ} \mathrm{C} \mathrm{min}{ }^{-1}$ from $20^{\circ} \mathrm{C}$ down to $-120^{\circ} \mathrm{C}$ ) immediately after the furnace reached $150^{\circ} \mathrm{C}$. All samples were scanned again from -120 to $150^{\circ} \mathrm{C}$ with a heating rate of $10^{\circ} \mathrm{C} \mathrm{min}{ }^{-1}$. The reported results are taken from the second heating runs of the experiments in order to avoid experimental artifacts arising from the previous thermal history and incomplete chemical reactions. In this study, the transition temperatures, endothermic peaks and exothermic peaks of the first and second heating runs are very close, with only the baseline slightly shifted.

Density measurements of the IPNs were carried out by use of a Micromeritics Accupyc 1330 gas pycnometer on finely cut samples at ambient temperature under a helium atmosphere. The samples were purged ten times by helium gas in order to ensure a consistent dry atmosphere during testing.

Water absorption of the IPNs was determined by immersing the cut IPN membranes in a beaker of water at $37^{\circ} \mathrm{C}$. The membranes, with a thickness of $1 \mathrm{~mm}$, were cut into circular disks by using a sharp-edged stainless steel die with inner diameter of $20 \mathrm{~mm}$. The samples were weighed every 10 min until they attained the maximum water content. The water absorption (WS) of the IPNs was calculated by

$W S=\left[\left(W_{\mathrm{s}}-W_{\mathrm{d}}\right) / W_{\mathrm{d}}\right] \times 100$

where $W_{\mathrm{s}}$ and $W_{\mathrm{d}}$ are the weights of the water-absorbed and the dry samples, respectively.

The determination of the equilibrium swelling ratio of the IPN samples followed the ASTM-D543 method using toluene as a solvent. The swelling ratio $(S R)$ was calculated by

$S R=\left(W-W_{\mathrm{o}}\right) / W_{\mathrm{o}} \times\left(d / d_{\mathrm{s}}\right)+1$

where $W$ is the weight of the swollen sample, $W_{\mathrm{o}}$ is the weight of the dry sample, $d$ is the density of the dry sample, and $d_{\mathrm{s}}$ is the density of toluene.

The mechanical properties were determined on an Instron TM-SM universal test unit at ambient temperature. The test procedure followed ASTM-D412 with a crosshead of $50 \mathrm{~mm} \mathrm{~min}^{-1}$. At least five specimens were tested per IPN composition.

Phase morphologies were studied on the cross-sectional surfaces of different IPNs using a Hitachi model S-550 scanning electron microscope. The samples were fractured in liquid nitrogen in order to preserve the sample morphology, and sputter-coated with gold prior to being viewed under the microscope.

\section{Results and discussion}

Differential scanning calorimetry (d.s.c.) curves of the HPU(PTMG1000)/PU(PPG1000) IPNs are shown in Fig. 3. Although there is no endothermic or exothermic peak detected, every sample exhibits a clear glass transition behaviour, seen as the slope change in the heat flow curve within the temperature range investigated and related to the relaxation process of the soft segments. For a given molecular weight, PPG molecules are shorter than PTMG molecules due to the methyl group substituents along their main chain, as seen in Fig. 2a. As a result of the shorter soft segment employed and the higher ratio of hard-to-soft segments, the glass transition temperature $T_{\mathrm{g}}$ of the PU(PPG1000) homopolymer is higher than that of the HPU(PTMG1000) homopolymer. Moreover, the glass transition of the HPU(PTMG1000) polymer is much weaker than that of the PU(PPG1000) polymer. This can be explained by their different network structures, as the HPU prepolymers with a functionality of four can form a network of much higher crosslinking density, which limits the degree of molecular chain motion at the glass transition, as schematically shown in Fig. 4a. The $T_{\mathrm{g}}$ values of the IPNs decrease as the HPU content increases, which indicates that the HPU(PTMG1000) polymer and the PU(PPG1000) polymer form compatible, one-phase IPNs, as seen in Table 2. This could result both from similar chemical structures and from appropriate matching reaction kinetics which allows the two components to form simultaneously interlocking networks. For up to a $50 \mathrm{wt} \%$ HPU content, the strength of the glass transitions of the IPNs is dominated by the PU.

Fig. 5 shows the d.s.c. curves of the HPU(PTMG1000)/ PU(PPG2000) IPNs up to a $50 \mathrm{wt} \% \mathrm{HPU}$ content. The $T_{\mathrm{g}}$ of the PU(PPG2000) homopolymer occurs at a much lower temperature $\left(-19.1^{\circ} \mathrm{C}\right)$ than that of its PPG1000 counterpart

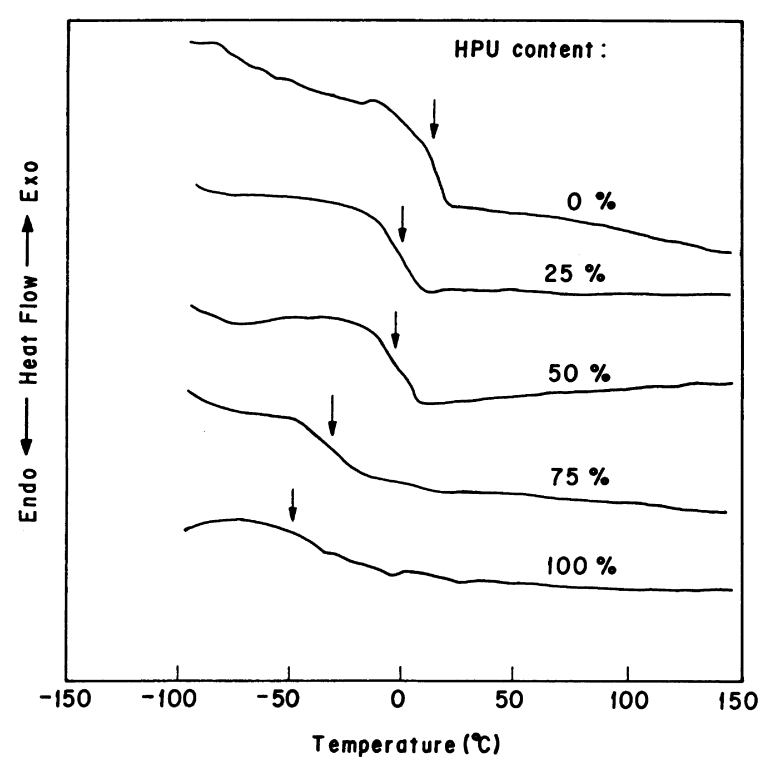

Fig. 3. D.s.c. thermograms of HPU(PTMG1000)/PU(PPG1000) IPNs. 

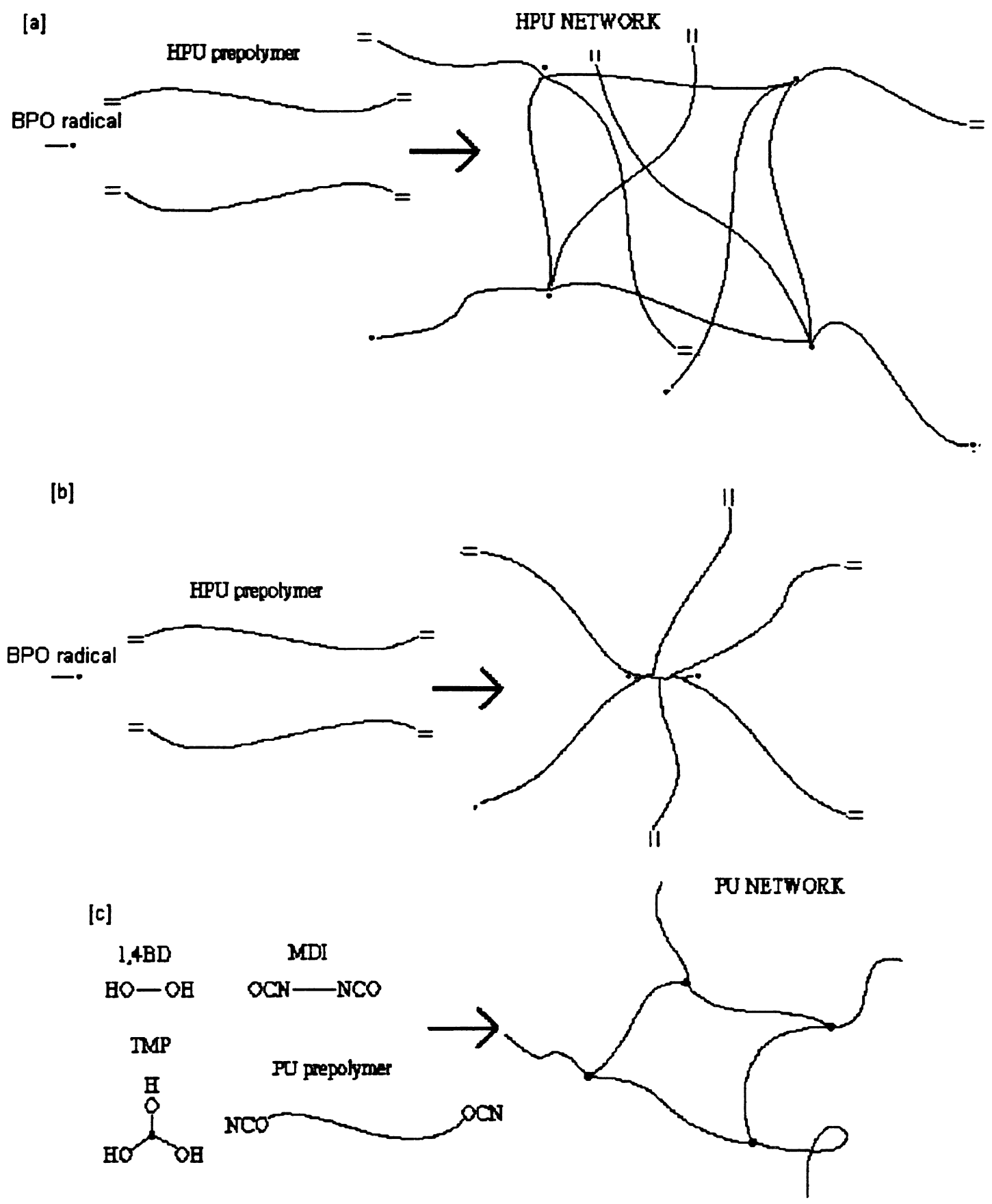

Fig. 4. Schematic structure of (a) the HPU network, (b) a possible HPU particle, and (c) the PU network.

$\left(17^{\circ} \mathrm{C}\right)$ as a higher molecular weight (more flexible) polyol was used. The $T_{\mathrm{g}}$ values of the HPU(PTMG1000)/ PU(PPG2000) IPNs, as shown in Table 2, appear to be close to that of the PU(PPG2000) polymer even at a $50 \mathrm{wt} \%$ HPU content. This indicates that the looser PU network of PPG2000 allows more effective interpenetration and leads to close packing of the HPU molecular chains compared with the HPU(PTMG1000)/PU(PPG1000) system. For samples of HPU(PTMG1000)/PU(PPG2000) containing more than $50 \mathrm{wt} \% \mathrm{HPU}$, the membranes could not be prepared with sufficient mechanical strength to allow their release intact from the dishes. This may result either from serious phase separation and/or the failure of the formation of a HPU network at these concentrations. It is possible that the addition of another component would dilute the concentration of double bonds and thus interrupt the formation of a continuous HPU network when the HPU is the major constituent. As the radical chain reaction tends to produce whole polymer almost instantaneously, the HPU is likely to form a structure similar to a 'star polymer', as shown in Fig. 4b. It would thus become a dispersed phase, allowing the IPN structure to form only at the interface [24]. 
Table 2

The $T_{\mathrm{g}}$ values for the various HPU/PU IPNs determined by d.s.c.

\begin{tabular}{|c|c|c|c|c|c|c|c|}
\hline & \multicolumn{7}{|c|}{ HPU content $(\%)$} \\
\hline & 0 & 12.5 & 25 & 37.5 & 50 & 75 & 100 \\
\hline \multicolumn{8}{|c|}{ HPU(PTMG100)/PU(PPG1000) IPNs (Fig. 3) } \\
\hline$T_{\mathrm{g}}\left({ }^{\circ} \mathrm{C}\right)$ & 17 & - & 0 & - & -3 & -31 & -48 \\
\hline \multicolumn{8}{|c|}{ HPU(PTMG1000)/PU(PPG2000) IPNs (Fig. 5) } \\
\hline$T_{\mathrm{g}}\left({ }^{\circ} \mathrm{C}\right)$ & -19 & -28 & 21 & -22 & -26 & - & -48 \\
\hline \multicolumn{8}{|c|}{ HPU(PTMG2000)/PU(PPG1000) IPNs (Fig. 6) } \\
\hline$T_{\mathrm{g}}^{\mathrm{a}}\left({ }^{\circ} \mathrm{C}\right)$ & - & -72 & -72 & -71 & -73 & - & -72 \\
\hline
\end{tabular}

${ }^{\text {a }}$ HPU(PTMG2000)-rich phase only.

By comparison, when using triol (TMP) as a crosslinking agent and diol (1,4-BD) as the chain extender, the PU network appears to be able to accommodate another network or particles due to the looser network structure, as shown in Fig. 4c.

Unlike the previous two IPN systems, IPNs of HPU(PTMG2000)/PU(PPG1000) exhibit a rather complicated thermal behaviour including both $T_{\mathrm{g}}$ values of their parent homopolymers (unless masked by other events), an exothermic peak and an endothermic peak, as shown in Fig. 6. With the addition of $12.5 \mathrm{wt} \%$ HPU(PTMG2000), there is (in addition to the $T_{\mathrm{g}}$ of the HPU(PTMG2000) polymer at $-72.4^{\circ} \mathrm{C}$ ) an exothermic peak which appears at $-28^{\circ} \mathrm{C}$ and an endothermic peak which appears at $29^{\circ} \mathrm{C}$. As the HPU content of the IPNs increases, the $T_{\mathrm{g}}$ of the HPU phase still remains at around $-72^{\circ} \mathrm{C}$ and the $T_{\mathrm{g}}$ of the PU phase at $17^{\circ} \mathrm{C}$ is masked by other peaks. In addition, the exothermic peak moves to higher temperatures and the endothermic one to lower temperatures as the HPU content increases, approaching those of the HPU homopolymer. Both the exothermic

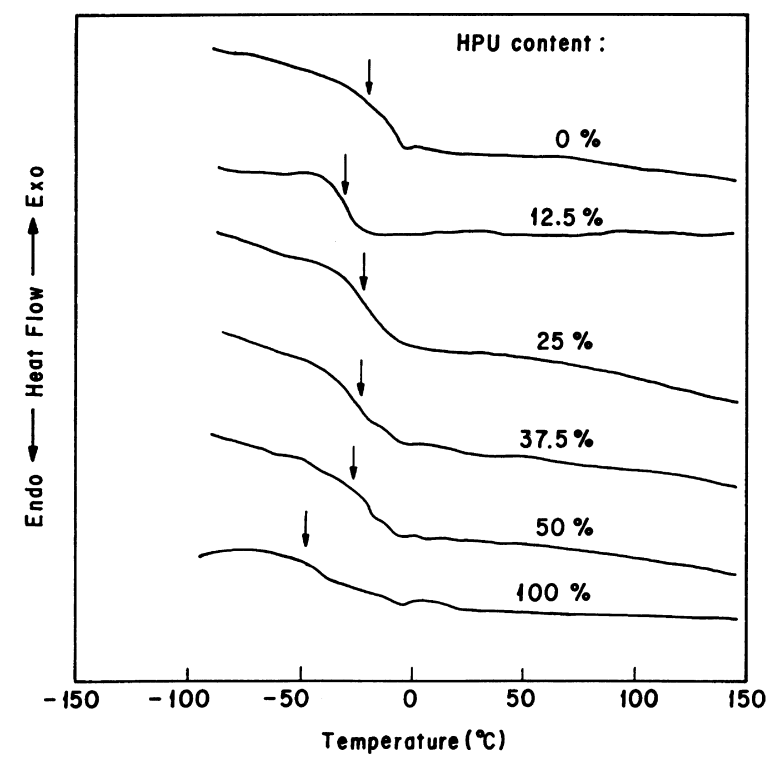

Fig. 5. D.s.c. thermograms of HPU(PTMG1000)/PU(PPG2000) IPNs.

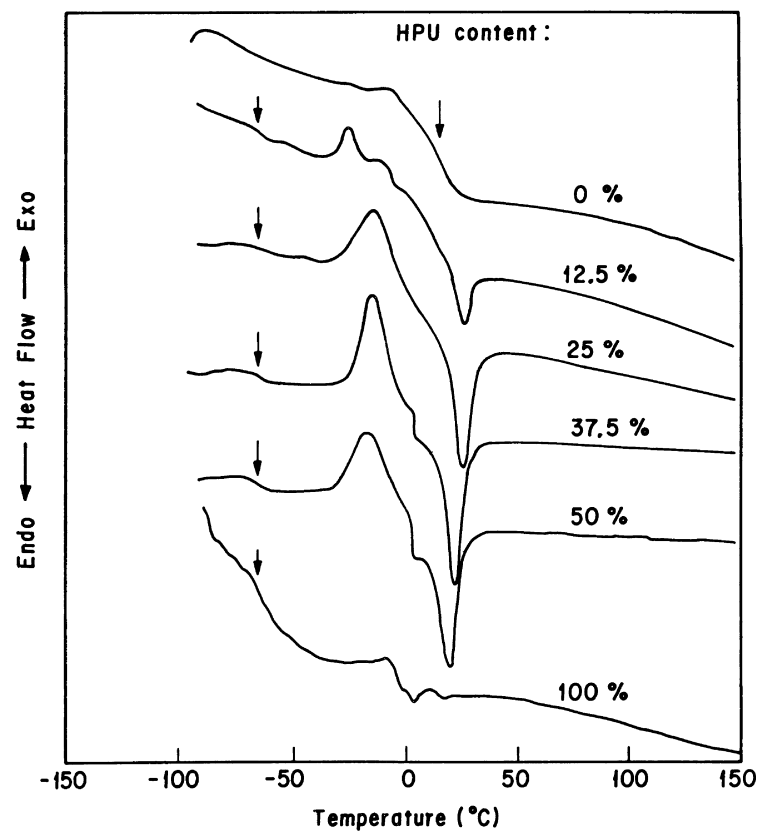

Fig. 6. D.s.c. thermograms of HPU(PTMG2000)/PU(PPG1000) IPNs.

and endothermic energies of the IPNs become greatest at a $37.5 \mathrm{wt} \%$ HPU content. It has been shown previously that the HPU(PTMG1000)/PU(PPG1000) system and the HPU(PTMG1000)/PU(PPG2000) system are compatible IPNs and the HPU(PTMG2000)/PU(PPG1000) system is an incompatible IPN, from results of dynamic mechanical analysis (DMA) [21]. As the peak temperatures are far lower than those relating to the hard urethane segment phase (above $150^{\circ} \mathrm{C}$ ), the recrystallization of soft segments of HPU could lead to exothermic peaks. The endothermic peaks are attributed to the melting of ordered HPU soft segments. Van Bogart et al. reported [25] that linear segmented PU with similar chemical components (MDI, PTMG2000 and 1,4-BD with an equivalent ratio of 2:1:1) showed an annealing-induced morphological change which displayed endothermic peaks at $25^{\circ} \mathrm{C}$ after being heat treated at various temperatures. In their study, under the same annealing treatment, the linear segmented PU based on PTMG1000 did not show these endothermic peaks, which appeared on the d.s.c. thermograms of linear segmented PU based on PTMG2000. This demonstrates that the presence of these endothermic peaks is a result of the ordering of the soft segments and is dependent on the length of the polyol component. As the neat HPU(PTMG2000) polymer does not exhibit these peaks to the same extent as the IPNs and linear segmented PUs with similar chemical structures [25], it indicates that the complete crosslinked HPU network produced by the HPU homopolymer restricts the ability of the soft segments to rearrange. It appears that in the IPNs, the loose end HPU chains do not react with others, allowing them to remain sufficiently mobile to self-order and show enhanced thermal behaviour. The longer, soft segments of the HPU(PTMG2000) network are probably responsible for 


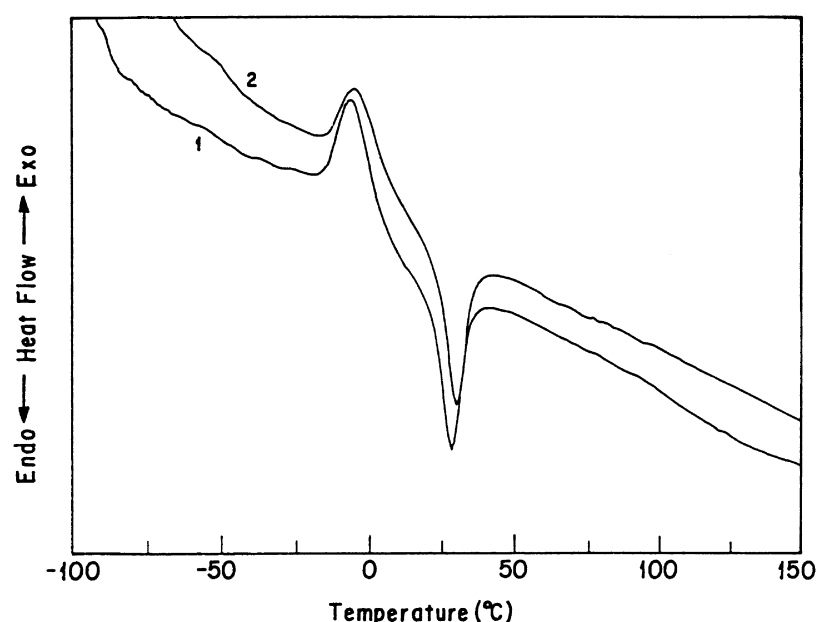

Fig. 7. D.s.c. curves of the HPU(PTMG2000)/PU(PPG1000)(37.5/62.5) IPN. First run and second run.

the incompatibility with the PU(PPG1000) network and facilitate the rearrangement of the soft segments. However, the precise nature of the ordering of the loose HPU chains, which can be carried out in such a short time during the d.s.c. measurements, remains unknown. All the d.s.c. curves reported are second heating runs for the same sample, as mentioned in the experimental section. Chemical reactions are ruled out due to the reversibility of this behaviour, as shown in Fig. 7, where the first heating run and the second heating run show almost the same behaviour, with only the baseline slightly shifted.

The composition dependence of the density of all the IPN systems is shown in Fig. 8. The two compatible systems, the HPU(PTMG1000)/PU(PPG1000) system and the HPU(PTMG1000)/PU(PPG2000) system, possess higher densities than that given by the rule of mixtures up to $50 \mathrm{wt} \%$ HPU. In a polymer blend where there is adhesion between the interface and no molecular mixing at the phase boundary, the density of the blend would be expected to follow the rule of mixtures. As a result, this positive deviation of the IPN densities can be attributed to intimate

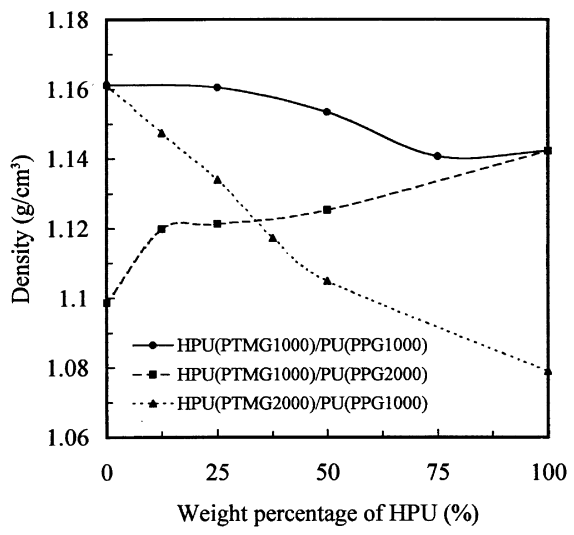

Fig. 8. The densities of various HPU/PU IPN systems. interpenetration and chain packing which leads to a reduction of the free volume. HPU(PTMG1000)/PU(PPG2000) IPNs show stronger positive density deviations than the HPU(PTMG1000)/PU(PPG1000) IPNs, which exhibit $T_{\mathrm{g}}$ values closer to that of the pure components with a higher $T_{\mathrm{g}}$ value from d.s.c. and DMA [21]. In contrast to these systems, the densities of the HPU(PTMG2000)/ PU(PPG1000) system show a negative deviation from the rule of mixtures and the deviation becomes larger with high HPU content. The results of the density measurements thus support the compatibility conclusions from d.s.c. observations regarding the compatibility of HPU/PU in different IPN systems, and prove to be a potent indication of chain mixing. The hypothesis that it is difficult for HPUs to form a continuous network if the interference of PUs is present, is thus also clear from density measurements. For compatible IPN systems, the positive deviation of the density reduces as the HPU content increases. In the case of incompatible IPN systems, the negative deviation of the density increases with increase in the HPU content. Even though the $75 \mathrm{wt} \%$ HPU content of HPU(PTMG1000)/ PU(PPG1000) IPN did not lead to gross phase separation according to the results from DMA [21] and d.s.c., its density is less than additive. The highly crosslinked HPU network may tend to exclude the other components, and poorer interpenetration results.

Fig. 9 shows the results of water absorption for various IPN systems. Water is believed to be primarily absorbed within the hard segment regions where the highly polar urethane group is much more hydrophilic than the soft segment. The neat PU network absorbed more water than HPU with the same molecular weight of polyol due to (i) a looser network structure; (ii) the more hydrophilic polyol used (order of hydrophilicity: poly(ethylene glycol) (PEG) > PPG > PTMG); and (iii) a greater hard-to-soft segment ratio. It is clear that the hard-to-soft segment ratio outweighs the other factors as the PU(PPG2000) homopolymer absorbed much less water than the PU(PPG1000) homopolymer. If water absorption was dominated simply by the degree of crosslinking of the network structure, the PU(PPG2000) homopolymer would have absorbed more water than the PU(PPG1000) homopolymer. The effect of the polyol molecular weight on the water absorption of HPUs, which is $2.21 \mathrm{~g}$ per $100 \mathrm{~g}$ for HPU(PTMG1000) and $2.29 \mathrm{~g}$ per $100 \mathrm{~g}$ for HPU(PTMG2000), is less significant. The water absorption of the looser HPU(PTMG2000) network is balanced by the higher hard-to-soft segment ratio of the HPU(PTMG1000) counterpart. The hydroxyl group of HEMA is strongly hydrophilic and HEMA has been used to produce hydrogels from its derivative materials [22,23]. In our study, all the hydroxyl groups of HEMA were consumed by reaction with isocyanate groups during the preparation of the HPU prepolymers. As a result, the incorporation of HEMA into the HPU polymer does not endow any additional hydrophilicity. Other than the HPU(PTMG1000)/PU(PPG1000) IPN with a 


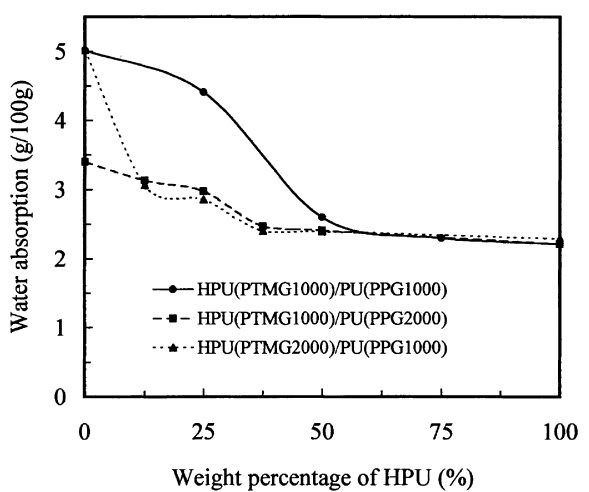

Fig. 9. The water absorption of various HPU/PU IPN systems.

$25 \mathrm{wt} \%$ HPU content, all IPNs show a strong negative deviation from their neat components since water absorption is hindered by the compact IPN structures.

It is of interest to study the IPN structure in a more fully swollen state, as these IPNs were formed as swollen gels by solution polymerization. A good solvent, toluene, was thus used to effectively swell the IPN structures. The results of swelling ratio tests on IPNs are shown in Fig. 10. The IPNs of HPU(PTMG1000)/PU(PPG1000) show a strong negative deviation of the swelling ratios from that of the neat component. The IPNs of HPU(PTMG1000)/ PU(PPG2000) also exhibit a negative deviation at low HPU content and approximate the rule of mixture average values at higher HPU content. Due to its incompatibility, the HPU(PTMG2000)/PU(PPG1000) system shows a positive deviation from additivity. This indicates that the compatible IPN systems make it harder for solvent to swell the network structure compared to the theoretical weighted average calculated from the swelling ratio of the homopolymers, due to the constrained molecular chains and lower free volume. The incompatible HPU(PTMG2000)/ PU(PPG1000) system, with its poor interpenetration and the presence of interfaces, makes it easier for solvent to enter via the interface between the phases. Because of the non-polar nature of toluene and the lack of interaction with the polar hard segment units, it is expected that toluene

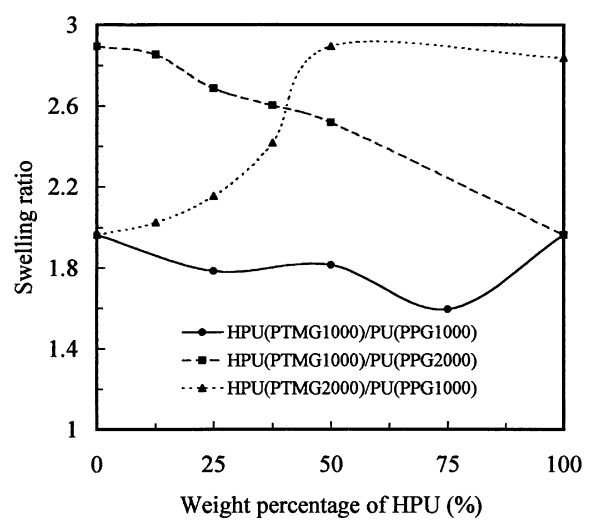

Fig. 10. The swelling ratios of various HPU/PU IPN systems.

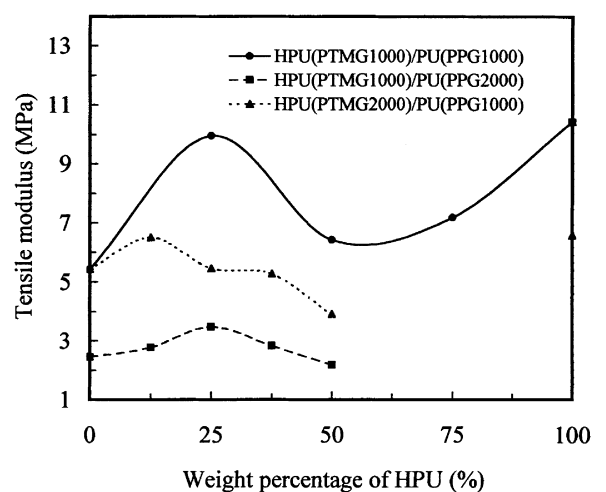

Fig. 11. The tensile moduli of various HPU/PU IPN systems.

swelling is more strongly dependent on compatibility and network structure than a polar solvent such as water.

The tensile moduli for various IPN systems are shown in Fig. 11. Neat HPU polymers have a larger tensile modulus than PUs with the same molecular weight of polyol due to their high crosslinking density. For the neat polymers, the tensile modulus decreases as the molecular weight of the polyol used increases. When the IPN system is a compatible one, the tensile modulus tends to increase with HPU content up to $25 \mathrm{wt} \%$, such as for the IPNs of HPU(PTMG1000)/ PU(PPG1000) and HPU(PTMG1000)/PU(PPG2000). It is worth pointing out that the tensile modulus of the IPN of HPU(PTMG1000)/PU(PPG1000) with a $25 \mathrm{wt} \%$ HPU content is almost the same as that of the neat HPU(PTMG1000) homopolymer, even though the major constituent of this IPN is PU homopolymer. By comparison, the tensile modulus of the incompatible HPU(PTMG2000)/ PU(PPG1000) IPNs fails to increase after the HPU content exceeds $12.5 \mathrm{wt} \%$.

Fig. 12 shows the tensile strengths of various IPN systems. The tensile strengths of all the IPN systems increase with HPU content up to $25 \mathrm{wt} \%$ and then decrease. Surprisingly, the phase-separated HPU(PTMG2000)/ PU(PPG1000) IPNs show a greater strength than the other compatible IPN systems. Note that both d.s.c. and DMA are capable of detecting domain sizes above 10-100 $\AA$ [26].

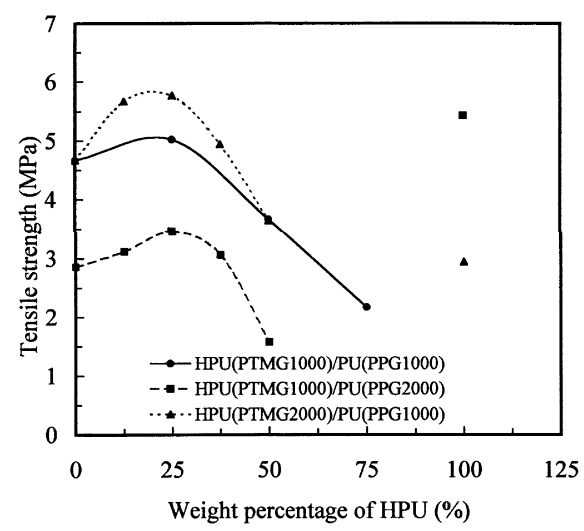

Fig. 12. The tensile strengths of various HPU/PU IPN systems. 


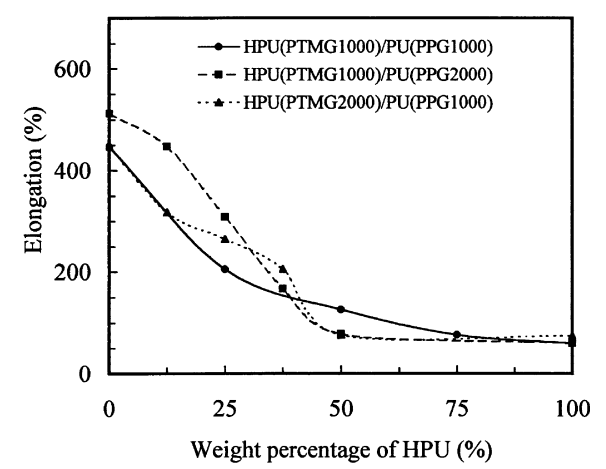

Fig. 13. The elongation at break of various HPU/PU IPN systems.

This scale of phase separation might still allow the formation of an effective IPN structure within the interface to interlock different phases. Moreover, the segregation of each phase assures the completion of the networks of HPU and PU, which might be interrupted by good mixing because these two components do not react with each other. Dilution effects of the different components may also reduce the reactivity. The influence of less interpenetration leads to a lower modulus, as seen in Fig. 11. However, the tensile strength measured at fracture indicates that a more thorough reaction of both the phases can compensate for the effect of less interpenetration. Longer PTMG2000 segments of the HPU could lead to more physical entanglements with the PU network and contribute to their ultimate strength. The effect of gross phase separation in the HPU(PTMG2000)/ PU(PPG1000) system can be seen in the rapid reduction of the tensile strength when the HPU content exceeds $25 \mathrm{wt} \%$.

The elongation of various IPN systems at failure is shown in Fig. 13. HPU(PTMG1000) and HPU(PTMG2000) show a rather brittle fracture compared with PU(PPG1000) and PU(PPG2000). The elongation of all the IPN systems displays a strong negative deviation from their components as increased interlocking of polymer segments from the IPN structure limits the extent of elongation at fracture. The elongation of IPNs with a $50 \mathrm{wt} \%$ HPU content or more is about $70 \%$ and is mainly dominated by the HPU component.

Observations of cryogenically fractured HPU(PTMG1000)/PU(PPG1000) IPN samples made with the aid of scanning electron microscopy (SEM) are illustrated in Fig. 14a-e. The morphology of neat HPU(PTMG1000) (Fig. 14a) and PU(PPG1000) (Fig. 14b) is smooth and indicative of a more homogeneous structure. The $25 \mathrm{wt} \%$ HPU IPN displays a rather rough surface feature, suggesting the existence of some finely dispersed HPU domains (Fig. 14c). When the HPU content is increased to $50 \mathrm{wt} \%$, the domain size of either the HPU or the PU seems to become larger with a dense and flat structure (Fig. 14d). There is a clear heterogeneous morphology with holes that are probably left by the dispersed PU-rich domains in the SEM photograph of 75 wt\% HPU IPN (Fig. 14e), despite this system having been shown to be compatible by DMA [21] and d.s.c.. Compared to the domain morphologies of polymer blends, which are usually incompatible due to a positive heat of mixing and negligible entropic changes on mixing, no clear domain structure is observed, indicating good bonding between different phases in these IPNs. For the case in which there is incompatibility, HPUs and PUs may form dispersed-continuous or co-continuous structures, depending on the composition of the prepolymers used, with interpenetration occurring only at the interfaces. The domain size and the effectiveness of intermolecular penetration will therefore determine the resultant performance. The HPU(PTMG2000) homopolymer also exhibits an homogeneous microscopic structure, as shown in Fig. 15a. Resulting from its inferior resistance to electron bombardment, some cracks appear on its SEM image but do not alter the overall topology. For incompatible IPNs of HPU(PTMG2000)/ PU(PPG1000), the morphologies show a layer-like structure with a rather large domain size, as seen in Fig. 15b and c. The fracture zone of these IPNs appears to extend beyond the surface, which is quite different from HPU(PTMG1000)/PU(PPG1000) IPNs. The enhanced tensile strength of this incompatible IPN system may result from the different fracture mechanism related to the morphology observed.

\section{Conclusions}

Interpenetrating polymer networks (IPNs) of 2-hydroxyethyl methacrylate terminated polyurethanes (HPUs) and polyurethanes (PUs) with different ether-type polyols, were studied. Analysis of the thermal behaviour of the IPNs using d.s.c. is simple in compatible IPN systems in which the glass transition $\left(T_{\mathrm{g}}\right)$ is the only feature detected. However, the d.s.c. curves in the incompatible IPN system show endothermic and exothermic peaks that are likely to be due to the rearrangement and ordering of the free end chains in the HPU network. The compatible IPN systems resulted in higher density values and a restrained swelling behaviour compared to their homopolymers. This results from high levels of interpenetration and molecular mixing, leading to a reduced free volume and a more compact network structure. Water absorption was related to a number of factors including the network structure, the hydrophilicity of the polyols used and the hard-to-soft segment ratio, with the last-mentioned appearing to be the dominant factor. The maximum tensile modulus occurred at a $25 \mathrm{wt} \% \mathrm{HPU}$ content for the compatible systems and a $12.5 \mathrm{wt} \% \mathrm{HPU}$ content for the incompatible system. The maximum tensile strength occurred at a $25 \mathrm{wt} \%$ HPU content for all the IPN systems. Morphological observations using the scanning electron microscope revealed different failure surfaces between the compatible and incompatible systems which is indicative of the different fracture mechanisms. 
[a]

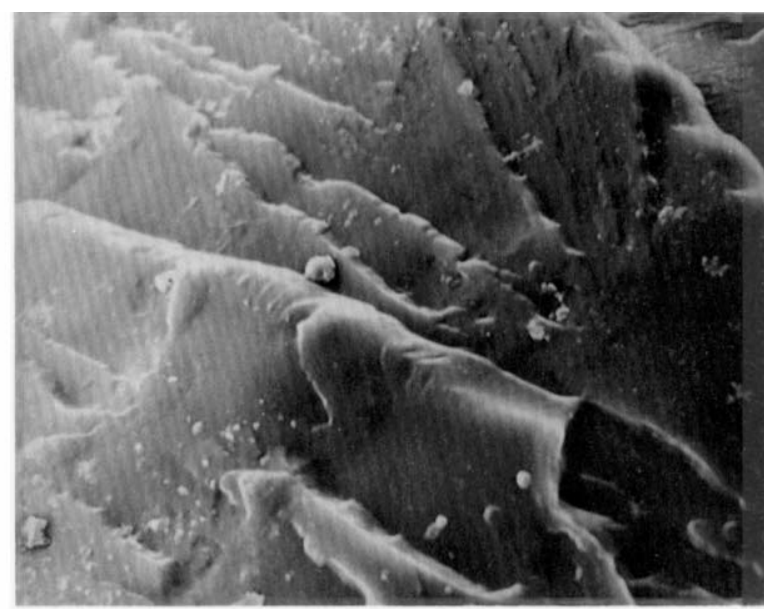

$\overline{5 \mu}$

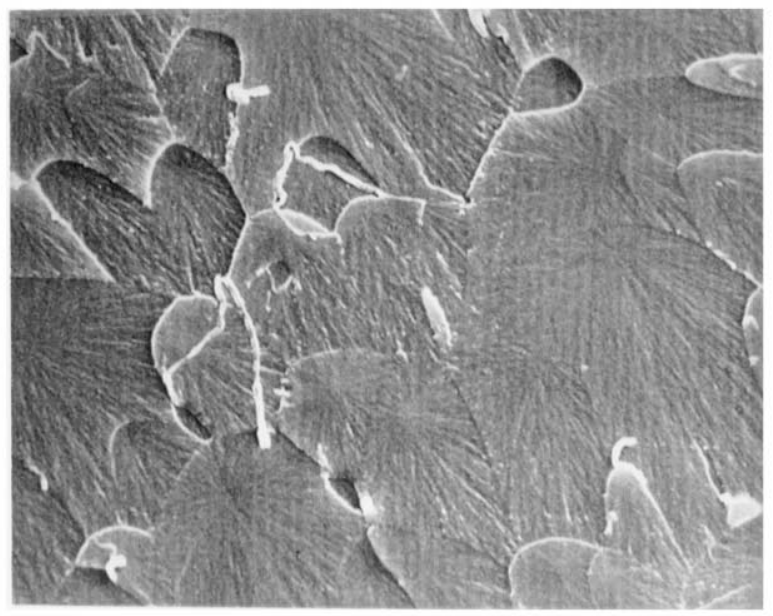

$\overline{5 \mu}$

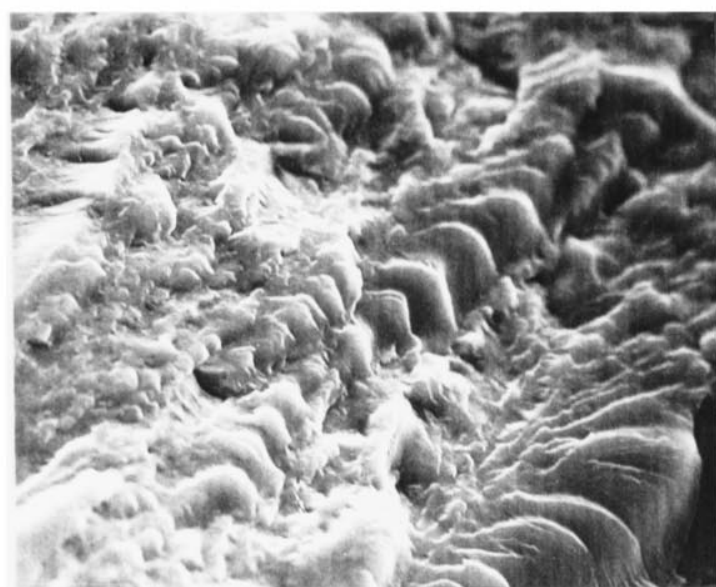

[c]

$$
\overline{5 \mu}
$$

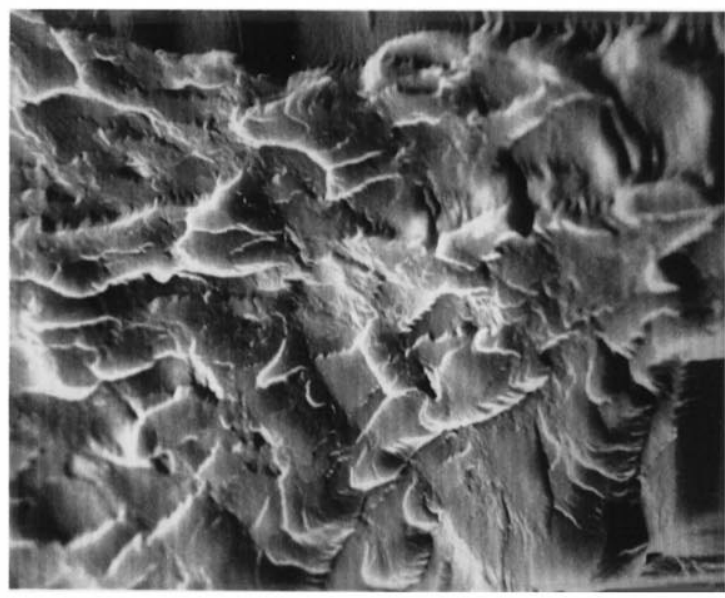

$\overline{5 \mu}$

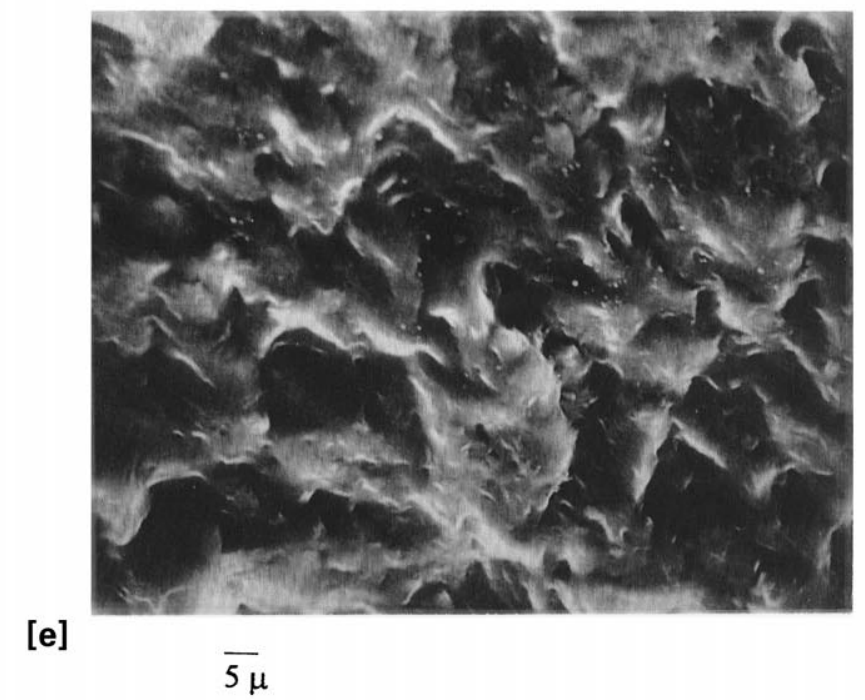

Fig. 14. Scanning electron micrographs of HPU(PTMG1000)/PU(PPG1000) IPNs. (a) HPU(PTMG1000) homopolymer; (b) PU(PPG1000) homopolymer; (c) IPN of HPU/PU $=25 / 75$; (d) IPN of HPU/PU $=50 / 50$; (e) IPN of HPU/PU $=75 / 25$. 


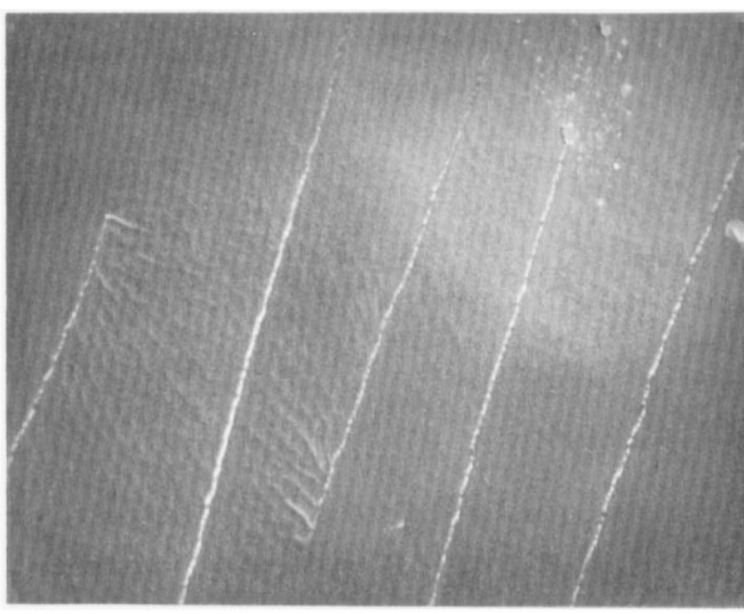

$\overline{5 \mu}$

[b]

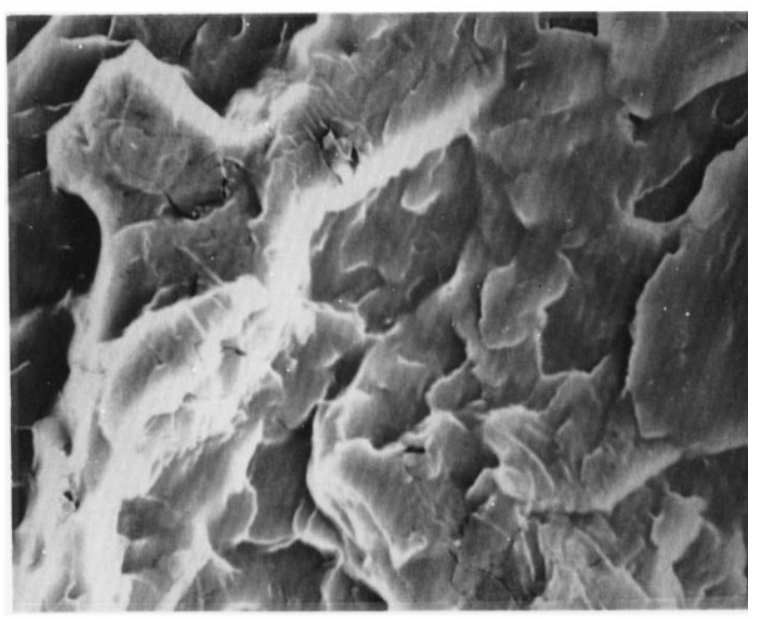

$\overline{5 \mu}$

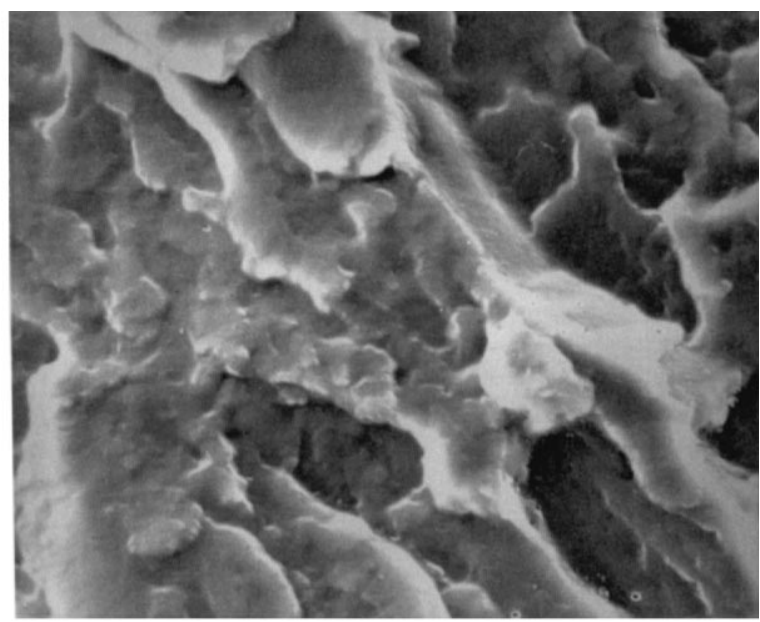

[c]

$\overline{5 \mu}$

Fig. 15. Scanning electron micrographs of HPU(PTMG2000)/ PPG(PPG1000 IPNs. (a) HPU(PTMG2000) homopolymer; (b) IPN of $\mathrm{HPU} / \mathrm{PPG}=25 / 75$; (c) $\mathrm{IPN}$ of $\mathrm{HPU} / \mathrm{PU}=50 / 50$.

\section{Acknowledgements}

Financial support from the National Science Council (NSC-80-0412-B002-180) is gratefully acknowledged.

\section{References}

[1] Sperling LH. Interpenetrating polymer networks and related materials. New York: Plenum Press, 1981, Chapter 1.

[2] Hsieh KH, Han JL. J Polym Sci B: Polym Phys 1989;28:623.

[3] Chou YC, Lee LJ. Polym Eng Sci 1995;35:976.

[4] Ali SAM, Hourston DJ, Manzoor K, Williams DF. J Appl Polym Sci 1995;55:733.

[5] Nayak P, Mishra DK, Parida D, Sahoo KC, Nanda M, Lenka S, Nayak PL. J Appl Polym Sci 1997;63:671.

[6] Yeo JK, Sperling LH, Thomas DA. Polym Eng Sci 1982;22:190.

[7] Yeo JK, Sperling LH, Thomas DA. Polymer 1983;24:307.

[8] Hourston DJ, Schafer FU. J Appl Polym Sci 1996;62:2025.

[9] Yao S. In: Klempner D, Frisch KC, editors. Advances in interpenetrating polymer network, vol. IV. Lancaster: Technomic, 1994:243.

[10] Li Y, Mao SF. J Appl Polym Sci 1996;61:2059.

[11] Hsieh KH, Liao DC, Chen CY, Chiu WY. Polym Adv Technol 1996;7:265

[12] Lee DS, Kwan SK, Kim SC. In: Klempner D, Frisch KC, editors. Advances in interpenetrating polymer network, vol. IV. Lancaster: Technomic, 1994:213.

[13] Hsieh KH, Tsai CC, Tseng SM. J Membr Sci 1990;49:341.

[14] Tsunoda S, Suzuki Y. In: Klempner D, Frisch KC, editors. Advances in interpenetrating polymer network, vol. II. Lancaster: Technomic, 1990:177.

[15] Buist JM, Gudgeon H. Advances in polyurethane technology. London: Elsevier, 1970.

[16] Hepburn C. Polyurethane elastomers, 2nd ed. New York: Elsevier, 1992.

[17] Oertel G. Polyurethane handbook, 2nd ed. New York: Hanser, 1993.

[18] Akutsu A. Artificial hearts, vol. 1. Tokyo: Springer-Verlag, 1985.

[19] Schauwecker HH, Gerlach T, Planck H, Bucherl ES. Artif Organs 1989;13:216.

[20] Pennings AT, Knol KE, Hoppen HJ. Colloid Polym Sci 1990;268:2.

[21] Liu CJ, Hsieh KH, Ho KS, Hsieh TT. J Biomed Mater Res 1997;34:261.

[22] Lai Y, Baccei LJ. J Appl Polym Sci 1991;42:2039.

[23] Lai Y, Baccei LJ. J Appl Polym Sci 1991;42:3137.

[24] Chou YC, Lee LJ. Polym Eng Sci 1994;34:1239.

[25] Van Bogart JWC, Bluemke DA, Cooper SL. Polymer 1981;22:1428.

[26] Utracki LA. Polymer alloys and blends: thermodynamics and rheology. New York: Hanser, 1989:64. 